\title{
Labyrinthe
}

25 | 2006 (3)

La Bande dessinée : ce qu'elle dit, ce qu'elle montre

\section{Sixième Congrès international d'histoire de la philosophie des sciences}

\author{
ENS rue d'Ulm, 14-18 juin 2006
}

Élodie Cassan

\section{OpenEdition}

\section{Journals}

Édition électronique

URL : http://journals.openedition.org/labyrinthe/1430

DOI : $10.4000 /$ labyrinthe. 1430

ISSN : 1950-6031

\section{Éditeur}

Hermann

\section{Édition imprimée}

Date de publication : 1 décembre 2006

Pagination : 143-145

ISBN : 2-9526131-2-5

Référence électronique

Élodie Cassan, «Sixième Congrès international d'histoire de la philosophie des sciences », Labyrinthe [En ligne], 25 | 2006 (3), mis en ligne le 28 mars 2010, consulté le 11 mai 2019. URL : http:// journals.openedition.org/labyrinthe/1430 ; DOI : 10.4000/labyrinthe.1430

Ce document a été généré automatiquement le 11 mai 2019.

Propriété intellectuelle 


\title{
Sixième Congrès international d'histoire de la philosophie des sciences
}

ENS rue d'Ulm, 14-18 juin 2006

\author{
Élodie Cassan
}

Depuis une vingtaine d'années, l'histoire de la philosophie des sciences suscite un intérêt grandissant au niveau international. Plusieurs ensembles de réalisations témoignent de cet intérêt ou contribuent à une meilleure connaissance de cette histoire. Tel est le cas de The International Society for the History of Philosophy of Science (Hopos), créée en 1992aux États-Unis ${ }^{1}$. Cette société savante entend la recherche sur l'histoire de la réflexion philosophique en science en un sens large, comprenant les sciences de la nature et les sciences de l'homme, la logique, la philosophie et les mathématiques. Incluant toutes les périodes de l'histoire des sciences, d'Aristote à nos jours, elle prend également en compte toutes les zones géographiques ainsi que les différentes méthodes de la science. Son approche généraliste de l'histoire des sciences à la fois contribue à la reconnaissance de celle-ci dans le champ des savoirs et permet la constitution d'un réseau international de chercheurs. Ceux-ci voient leurs interrelations facilitées grâce à une liste de discussion électronique très active, dont le modérateur n'est autre que le philosophe des sciences américain Don Howard ${ }^{2}$. En outre, ils sont informés sur l'actualité de la recherche en histoire des sciences grâce à un bulletin électronique bi-annuel. Enfin, ils sont invités à se rencontrer lors de congrès internationaux biennaux.

2 Le dernier en date s'est tenu à l'École normale supérieure à Paris en juin dernier, en collaboration avec la Société de philosophie des sciences ${ }^{3}$. Accueillir des travaux portant sur l'histoire de la philosophie des sciences, toutes époques et toutes approches disciplinaires confondues, n'allait pas sans une organisation conséquente. Étaient donc prévus quatre jours et demi de conférences, marqués par cinq sessions tenues en parallèle, et divisées en trois symposia, ensemble de trois ou quatre communications sur un thème donné, et en deux séances d'interventions individuelles. Chacune de ces journées était orientée par une diversité de fils conducteurs thématiques. 
Dans ces conditions, proposer un panorama des contributions présentées et une liste de leurs auteurs n'est pas envisageable. On peut néanmoins indiquer que trois objets et une question ont retenu l'attention des participants. Ces objets sont la science de l'époque moderne, soit la révolution scientifique des XVIe et XVIIe siècles, les sciences de la vie du XIXe siècle et la philosophie de la physique du XXe siècle. La question est celle de savoir comment l'épistémologie se constitue, et ce qu'elle peut et doit dire de la science.

D'autre part, on peut rappeler les trois points majeurs qui se sont dégagés des interventions sur la science de l'époque moderne. D'abord, ces communications allaient dans un même sens. Discutant une lecture de l'histoire des sciences à la manière d'Alexandre Koyré, elles ne réduisaient pas les sciences des XVIe et XVIIe siècles, en l'occurrence essentiellement les sciences de la nature, à des corps de doctrine purement rationalistes. Partant de l'idée théorisée par Paolo Rossi ${ }^{4}$, et selon laquelle l'on peut distinguer deux figures du scientifique, l'une rationaliste, d'inspiration cartésienne, ramenant la science à des déductions a priori, l'autre empiriste, d'inspiration baconienne, revenant à consulter la nature de façon athéorique, elles s'intéressaient surtout à la science empirique. Dans cette perspective, Dana Jalobeanu de l'université Vasile Goldis, Arad, Roumanie, a montré que les débats sur la nature de la collision, qui se tiennent à la Royal Society de Londres dans la seconde moitié du XVIIe siècle procèdent d'une remise en cause des fondements théoriques de la physique cartésienne au profit de la physique empiriste de Gassendi.

Ensuite, la question de la mécanisation de la nature a été abordée de façon nouvelle. Un symposium organisé par Simone Mazauric (Nancy II) a cherché moins à valider la thèse selon laquelle la mécanisation de la nature, telle qu'elle a été proposée par Descartes notamment, est le cœur de la révolution scientifique du XVIIe siècle, qu'à en interroger la portée. La question posée était celle de savoir en quel sens et dans quelle mesure les savants européens de la seconde moitié du XVIIe siècle ont effectivement et unanimement effectué cette mécanisation, dans sa double dimension polémique (contre la représentation aristotélicienne de la nature) et affirmative (réduction de la matière à la grandeur, à la figure et au mouvement). Différents intervenants ont alors fait voir comment l'hypothèse mécaniste est mise à distance par quelques philosophes et savants européens après 1650. En ce sens, Philippe Hamou (Paris X) a souligné le caractère problématique sur le plan épistémologique de cette hypothèse chez Locke. Daniel Garber (Princeton) a quant à lui mis en lumière le fait qu'il faut que le jeune Leibniz rencontre Spinoza pour en venir à la philosophie mécanique et pour la réformer.

6 Enfin, l'intérêt pour les disciplines scientifiques dans leur configuration historique ne dispensait pas d'une réflexion sur la notion même de discipline. Ce point était au cœur d'un symposium organisé par Marco Panza du CNRS. Afin d'établir la philosophie des mathématiques comme discipline, cette session souhaitait montrer qu'à l'âge classique la philosophie des mathématiques et les mathématiques ont une histoire différente. En ce sens, David Rabouin (CNRS) a montré que la mathesis universalis, concept qui circule dès le début du XVIe siècle, ne saurait être reconstruite comme une simple projection méthodologique, voire fondationaliste des mathématiques vivantes.

7 Privilégiant l'empirisme par rapport au rationalisme, analysant la physique de la fin du XVIIe siècle, interrogeant les mathématiques au XVIe, Hopos 2006 a ainsi mis en lumière la richesse de la philosophie des sciences de l'époque moderne et, dans ce domaine, fait apparaître le non-sens qu'il y aurait à la réduire à la seule figure de Descartes. 


\section{NOTES}

1. http://cas.imkc.edu/scistud:hopos

2. Pour toute question concernant cette liste, il convient d'écrire à dhoward1[at]nd.edu

3. Rue d'Ulm, du 14 au 18 juin 2006.

4. Aux origines de la science moderne, traduit de l'italien par Patrick Vighetti, Paris, Seuil, 1999, p. 163-164. 